EDITORIAL

\title{
¿Existe realmente la adicción al sexo?
}

\section{Does really sex addiction exist?}

I ENRIQUe ECHEBURÚA
Universidad del País Vasco UPV/EHU
Cibersam

\author{
Enviar correspondencia a: \\ Enrique Echeburúa. \\ Universidad del País Vasco UPV/EHU. \\ Facultad de Psicología. \\ Avda. de Tolosa, 70. 20018 San Sebastián (España). \\ enrique.echeburua@ehu.es
}

\section{Resumen}

Se ha propuesto el trastorno de hipersexualidad como una nueva categoría diagnóstica para el DSM-V. Este trastorno se caracteriza por la frecuencia y la intensidad de las fantasías sexuales, la activación psicofisiológica, el ansia y las conductas sexuales, todo ello asociado a un componente de impulsividad. Las conductas excesivas de tipo apetitivo, incluyendo la hipersexualidad, pueden considerarse como adicciones conductuales. La adicción al sexo se aplica a personas que muestran conductas parafilicas o no parafílicas relacionadas con situaciones de riesgo, con una escalada en el tipo de conductas sexuales (tolerancia), con una pérdida de control y con una consecuencias psicosociales negativas, tales como embarazos no deseados, ruptura de pareja, problemas económicos/ laborales y enfermedades de trasnmisión sexual, incluyendo el sida. Las conductas más habituales implicadas en la adicción al sexo son las fantasias sexuales, la masturbación compulsiva, la pornografía, el cibersexo, el voyeurismo, el sexo anónimo y las parejas múltiples. Las conductas sexuales tienen como objetivo reducir la ansiedad y otros afectos disfóricos (por ejemplo, la vergüenza y la depresión). La adicción al sexo presenta una comorbilidad con otros trastornos del Eje I (sobre todo, con trastornos de ansiedad, depresión, dependencia de sustancias e hiperactividad). Todavia hay muchas lagunas en el conocimiento del curso clínico, de los factores de riesgo y de la historia familiar y se carece de datos sobre la adición al sexo en mujeres.

Palabras clave: Trastorno de hipersexualidad, adicción al sexo, parafilias, comorbilidad, DSM-V.

\section{Abstract}

Hypersexual Disorder has been proposed as a new psychiatric disorder for DSM-V, characterized by an increased frecuency and intensity of sexually motivated fantasies, arousal, urges, and enacted behavior in association with an impulsivity component. Excesssive appetitive and consummatory behaviors, including hypersexuality, can become a nonchemical addiction. Sexual addiction afflicts people having paraphilic or nonparaphilic behaviors associated with progressive risk-taking sexual behaviors, escalation or progression of sexual behaviors (tolerance), loss of control and significant adverse psychosocial consequences, such as unplanned pregnancy, pair-bond dysfunction, marital separation, financial problems and sexually transmitted diseases including HIV. The most common behaviors involved in sexual addiction are fantasy sex, compulsive masturbation, pornography, cybersex, voyeuristic sex, anonymous sex and multiple sexual partners. These behaviors are intended to reduce anxiety and other dysphoric affects (e.g., shame and depression). Axis I psychiatric diagnosis, especially mood disorders, psychoactive substance abuse disorders and attention deficit hyperactivity disorders, are common comorbid disorders with sexual addiction. There are significant gaps in the current scientific knowledge base regarding the clinical course, development risk factors and family history and data on women with sexual addiction are lacking..

Key words: Hypersexual disorder, sexual addiction, paraphilias, comorbidity, DSM-V. 
a incorporación al próximo DSM-V de una nueva categoría diagnóstica (el trastorno de hipersexualidad), con

algunas características definitorias propias de las conductas adictivas (tabla 1), ha replanteado la posible existencia de una adicción al sexo (Kafka, 2010), que, por otra parte, es objeto de estudio por parte de una revista monográfica (Sexual Addiction \& Compulsivity), publicada desde 1994.

\section{Tabla 1. Criterios diagnósticos para el Trastorno de Hipersexualidad según el DSM-V}

A. Durante al menos seis meses, fantasias sexuales recurrentes e intensas y deseo sexual apremiante, asi como conductas sexuales asociadas a cuatro o más de los siguientes cinco criterios:

(1) Cantidad de tiempo excesiva invertida en fantasias y deseos sexuales, así como en la planificación y realización de conductas sexuales.

(2) Fantasias, deseos y conductas sexuales repetidas en respuesta a estados de ánimo disfóricos (p. ej., ansiedad, depresión, aburrimiento, irritabilidad).

(3) Fantasias, deseos y conductas sexuales repetidas en respuesta a situaciones vitales estresantes.

(4) Intentos persistentes pero infructuosos para controlar o reducir significativamente las fantasías, deseos y conductas sexuales.

(5) Implicación repetida en conductas sexuales ignorando el riesgo físico, psíquico o emocional que pueda suponer para si mismo o para otras personas.

B. La frecuencia o intensidad de las fantasias, deseos y conductas sexuales provocan malestar clínicamente significativo o deterioro social, laboral o de otras áreas importantes de la actividad de la persona.

C. Las fantasías, deseos y conductas sexuales no son debidos a los efectos fisiológicos directos de una sustancia (p. ej., una droga, un medicamento) ni a episodios maníacos.

D. El sujeto es mayor de 18 años.

Especifiquese si el problema planteado es:

Masturbación, pornografía, conducta sexual con adultos que consienten, cibersexo, sexo telefónico, clubs de "striptease", otros problemas.

Especificar si:

En remisión

Especifiquese si:

En remisión (no hay malestar emocional, deterioro o conducta recurrente en un ambiente no controlado): Duración de la remisión en meses:

En un ambiente controlado

La adicción al sexo, descrita inicialmente por Carnes en 1983 y por Goodman (1997), supone el desarrollo de conductas sexuales irrefrenables utilizadas para producir autogratificación. Se trata de una cadena de conductas estreotipadas más allá del control del sujeto, que interfieren en su vida cotidiana, pero que no se puede dejar de practicar a pesar de los aspectos negativos implicados: sentimientos de culpa, inminente ruptura de pareja, daño ocasionado a la familia, temor a enfermedades de transmisión sexual, problemas económicos o amenaza de pérdida de empleo. La adicción al sexo implica un conjunto recurrente de conductas, pensamientos y fantasías, activación fisiológica y ansia por llevar a cabo conductas sexuales. En este sentido el adicto al sexo es como un alcohólico que se caracteriza por una hipersexualidad que, al no saciarse, está permanentemente presente en el sujeto, que prescinde por completo de la ternura en la relación íntima y que manifiesta una pérdida de control (Echeburúa, 1999).

La conducta habitual del sexoadicto parte de un impulso incontrolado dirigido a la práctica sexual física y anónima, en un acto breve, frecuentemente poco satisfactorio, que se repite con intervalos variables siempre cortos -entre algunas horas y escasos dias-, con parejas distintas y sin reparar en los perjuicios de toda índole que tal conducta ocasiona a uno mismo y a su familia. Se trata de un hábito sexual desvinculado de todo propósito de comunicación y sin el menor atisbo de vivencia amorosa (Alonso-Fernández, 1996). En general, el tipo de relación sexual que no tiene connotaciones afectivas, es decir, el que no implica un componente de afecto o ternura, cuenta con un mayor potencial adictivo.

La sexoadicción afecta más a hombres que a mujeres. Los datos epidemiológicos disponibles son aún muy provisionales. Según la Society for the Advancement of Sexual Health (2012), entre el 3\% y el 5\% de las personas en la sociedad norteamericana pueden ser consideradas como sexoadictas. Según un estudio epidemiológico llevado a cabo en Suecia (Langström y Hanson, 2006), el $12,1 \%$ de los hombres y el $6,8 \%$ de las mujeres podían calificarse como hipersexuales. Además, es una adicción invisible: a diferencia de otras adicciones químicas, no se nota ni en la cara ni en el exterior de la persona ni queda reflejada en los análisis de sangre u orina.

Como ocurre también en otras conductas adictivas (Echeburúa y Corral, 2010; Potenza, 2006), lo que lleva a la búsqueda de la relación sexual es más el alivio del malestar que la búsqueda del bienestar. La adicción al sexo, a diferencia de la hipersexualidad normal, se caracteriza porque la conducta no es intrínsecamente de índole sexual, sino que con ella se trata de reducir la ansiedad o de escapar de un desasosiego interno. El sexo se convierte en un remedio para reducir el malestar emocional y la actividad sexual en algo morboso y obsesivo. De este modo, se utiliza el sexo como una estrategia de afrontamiento. Si se está eufórico, el cuerpo pide celebrarlo. Si se está desanimado, es una forma de animarse (Bancroft, Jannsen, Carnes, Strong, Goodrich y Long, 2004; Reid, Harper y Anderson, 2009).

A diferencia de otras adicciones, la sexual puede adoptar múltiples formas: desde la masturbación compulsiva, las relaciones promiscuas breves con múltiples parejas heterosexuales u homosexuales, los encuentros sexuales con personas desconocidas o la frecuentación habitual de prostíbulos, hasta el uso de pornografía (vídeos, revistas, ciberporno) o de líneas telefónicas eróticas, en donde se intentan satisfacer fantasías sexuales de toda índole. Si bien todas las parafilias (pedofilia, exhibicionismo, travestismo, prácticas sadomasoquistas, etcétera) cumplen criterios de adicción 
sexual, esta no tiene por qué ser necesariamente parafílica. Así, hay manifestaciones no parafílicas de esta hipersexualidad anómala: autoerotismo compulsivo, búsqueda ansiosa de relaciones sucesivas con múltiples parejas o una conducta hipersexual y obsesiva en una única relación.

La llegada de Internet no ha hecho más que complicar el panorama. No se trata solo de las webs del ciberporno, sino de la práctica desinhibida del sexo con personas desconocidas, que está más al alcance de la mano gracias a la facilidad de contactos que se establecen en la Red. Se trata en muchos casos de relaciones indiscriminadas de tipo kleenex (de usar y tirar), que responden a una necesidad percibida como desbordante e incontrolable por parte del sujeto.

El sexo por Internet, que ofrece todas las variantes imaginables, tiene una demanda creciente por su gran capacidad para producir enganche adictivo. Así, el cibersexo triunfa por ser accesible, anónimo y asequible, por fomentar la fantasía y por ser una vía de escape para las personas más tímidas. Son síntomas de adicción: a) pasar muchas horas en los chats eróticos o buscando cibersexo; b) buscar personas anónimas para desarrollar fantasías sexuales que normalmente no se realizan en la vida real; c) alternar el cibersexo con los teléfonos eróticos; d) masturbarse mientras se está en un chat erótico; y e) preferir obtener el placer sexual en Internet antes que en la relación con la propia pareja.

El comportamiento sexual adictivo se gesta, en la mayoría de los casos, en la mente, donde las fantasías sexuales y los pensamientos eróticos se convierten en la válvula de escape de los problemas laborales, las relaciones rotas, la baja autoestima o la insatisfacción personal. Otras veces es el alcohol o alguna otra droga estimulante, como la cocaína, lo que pone en marcha el circuito adictivo. En cierto modo, la caña puede llevar a la raya y la raya a la conducta sexual. La cocaína libera la dopamina, justamente el mismo neurotransmisor que activa el deseo sexual (Goodman, 2008).

Los signos alertadores de la sexoadicción son múltiples: fantasias sexuales reiteradas que alejan al sujeto de la relación de pareja; actos sexuales anónimos, múltiples y breves; sexualidad habitualmente comprada (prostitutas, teléfono erótico); práctica reiterada del voyeurismo (mirar a los vecinos por las ventanas esperando su desnudo, vigilar parejas); conductas de acoso sexual, etcétera.

En los adictos al sexo muchos estímulos no sexuales adquieren un valor erótico por un proceso de condicionamiento. Así, para ciertos adictos lo importante es el ritual: la conducción del coche durante horas hasta dar con la prostituta buscada o la vigilancia paciente detrás de una ventana a oscuras a la espera de espiar una relación sexual en la casa de enfrente. Todas estas conductas previas generan una gran excitación al sexoadicto.

\section{Consecuencias}

La dependencia al sexo tiene su reflejo en la aparición de un síndrome de abstinencia caracterizado por nerviosismo, irritabilidad, ansiedad, dolores de cabeza, temblores e insom- nio. Asimismo, al habituarse el sujeto a los estímulos, estos necesitan ser cada vez más excitantes para experimentar el goce inicial (tolerancia) (Echeburúa, 1999).

El adicto pierde varias horas todos los días en la búsqueda del objetivo sexual en una vorágine de sexo sin control. El orgasmo genera insatisfacción, pero esta lleva, a su vez, a la búsqueda compulsiva del placer. Como en el caso del resto de las adicciones, la sexoadicción conlleva consecuencias negativas en la vida cotidiana: desmoronamiento de la autoestima, pérdida de la pareja, riesgo de contraer sida y enfermedades de transmisión sexual, problemas legales derivados del acoso sexual, pensamientos obsesivos en relación con el sexo e incluso intentos de suicidio. Además, en las personas afectadas es habitual dejar de lado el cuidado del aspecto físico y de la salud, así como las actividades sociales, laborales o familiares (Kalichman, Cherry, Cain, Pope y Kalichman, 2005).

Esta adicción genera un grado alto de sufrimiento y autodestrucción. En general, se trata de una adicción más vergonzante y menos confesada que otras, lo que lleva a la soledad y comporta un intenso sentimiento de culpa. De este modo, en una espiral sin fin, el sexo cada vez va ocupando más espacio en la vida y en el pensamiento del sujeto y acaba por destruirlo (divorcio, ruina, suicidio) (Miner, Coleman, Center, Ross y Rosser, 2007; Nelson y Oehlert, 2008).

En resumen, la vida sexual se vive en secreto y con culpa. Si hay ausencia de mentiras, no se da una verdadera adicción. Se trata de personas capaces de llevar una doble vida. Una vez establecida la adicción y con la conciencia de la pérdida de libertad, los sexoadictos sufren ansiedad, dejan de sentir placer y viven su situación con vergüenza, soledad y pérdida de autoestima (Kafka, 2010).

\section{Etiología, estatus clínico y comorbilidad}

Las causas de este trastorno no están del todo claras. En la infancia de algunos sexoadictos puede haber abusos 0 traumas sexuales (que los afectados frecuentemente suelen negar o desconocer). En otras personas, sin embargo, es el fracaso de una relación afectiva en la vida adulta, vivido de forma muy dramática, lo que actúa como desencadenante. Otras veces se trata de discapacitados mentales o de enfermos mentales severos que se implican en este tipo de conductas estereotipadas.

Como en el resto de las adicciones (Echeburúa, Bravo de Medina y Aizpiri, 2005, 2007, 2009), hay algunas dimensiones de personalidad que hacen a la persona más vulnerable a la sexoadicción, como es el caso de la impulsividad acentuada, la búsqueda de sensaciones/riesgos o los rasgos obsesivos (Janssen, Goodrich, Petrocelli y Bancroft, 2009). En general, la insatisfacción, las carencias afectivas y las sustancias adictivas (alcohol y cocaína) están detrás de un síndrome capaz de destruir a quien lo padece.

La hipersexualidad ha sido catalogada también como un trastorno del control de los impulsos, en donde la respuesta 
inmediata a un ansia sexual no toma en consideración las consecuencias de la conducta (Bancroft et al., 2004; Janssen et al., 2009), o incluso una compulsión sexual, sobre todo si se tiene en cuenta que muchas conductas sexuales repetitivas suponen un intento de reducir la ansiedad, el estrés y otros estados de ánimo disfóricos (la culpa o la tristeza) (Nelson y Oehlert, 2008). De este modo, la obsesión sexual supone la presencia de pensamientos o fantasías sexuales invasivos que intentan controlarse con la conducta sexual compulsiva (Kafka, 2010). Cabe hipotetizar, por analogía con la dependencia de sustancias adictivas, que la hipersesexualidad está asociada con la impulsividad en las primeras fases del trastorno y con la compulsividad en las fases más avanzadas, cuando se establecen unas claras consecuencias negativas a nivel personal (imposibilidad de frenar la escalada) y familiar/social (Koob, 2009).

Entre las personas de riesgo se encuentran aquellas que cuentan con una baja autoestima, que muestran una insatisfacción con su imagen corporal, que presentan algún tipo de disfunciones sexuales o que tienen un historial insatisfactorio de relaciones de pareja.

La adicción al sexo puede estar ligada a los problemas de ansiedad o a la depresión (más en el caso de las mujeres), con el consiguiente riesgo de suicidio, a problemas en el control de los impulsos, a diversos trastornos de la personalidad (histriónico, narcisista, paranoide u obsesivo-compulsivo especialmente) o al consumo abusivo de alcohol y drogas (más en el caso de los hombres), sobre todo cocaína (Krueger y Kaplan, 2000; Raymond, Coleman y Miner, 2003; Reid et al., 2009). En este último caso el alcohol es en muchas ocasiones el interruptor que pone en marcha la cadena de conductas adictivas. Asimismo en algunos estudios el trastorno por déficit de atención con hiperactividad (subtipo inatento) ha resultado ser comórbido con la adicción al sexo (Blankenship y Laaser, 2004; Reid, 2007).

Las personas sexoadictas pueden implicarse en otras conductas de riesgo, tales como fumar, abusar del alcohol o de las drogas o jugar en exceso a juegos de apuestas (Langström y Hanson, 2006).

\section{Reflexiones finales}

Por primera vez en las clasificaciones psicopatológicas el DSM-V incorpora la categoría diagnóstica de trastorno de hipersexualidad, si bien los criterios diagnósticos establecidos están aún a falta de una validación empírica. Como se puede observar en la tabla 1, se requiere para llevar a cabo este diagnóstico la presencia de, al menos, cuatro de los cinco criterios del Apartado A. Esta exigencia tiene como objetivo minimizar la tasa de falsos positivos con este diagnóstico $y$, en último término, evitar la psicopatologización de variantes de la conducta sexual existentes en la vida cotidiana. Este trastorno no es consistente con su conceptualización como un trastorno del espectro obsesivo-compulsivo, sino con el de una adicción no-química (Kafka, 2010).
La adicción al sexo no es ni puede convertirse en una categoría moral, sino psicopatológica. La promiscuidad sexual, las relaciones homosexuales, el cambio frecuente de pareja pueden suscitar un reproche moral en algunos sectores de la sociedad, pero no constituyen por sí una categoría psicopatológica ni se debe recurrir a ella para justificar dicho reproche. Los debates morales deben mantenerse en el terreno de la moral. Solo se puede hablar de adicción al sexo cuando hay una pérdida de libertad por parte del sujeto afectado (recurre a ciertas conductas sexuales a su pesar y sin ser capaz de evitarlas), cuando hay ansia y fantasías sexuales recurrentes, cuando el sexo se convierte en el centro de la vida de una persona y cuando supone una interferencia grave en su vida cotidiana (malestar emocional y consecuencias negativas) (Kafka, 2010).

La sexoadicción está relacionada tanto con conductas parafílicas como no parafílicas. El sexoadicto confunde el amor con el éxtasis sexual. El sexo es la única vía para conseguir una gratificación personal y afectiva. El placer de la conducta sexual se puede convertir en una fuente permante de sufrimiento cuando una persona se convierte en un esclavo del sexo y pierde la familia, la autoestima y el dinero en la búsqueda infinita de sexo. Es más, el sexoadicto llega a cosificar a las personas y a considerarlas solo como objetos sexuales. Cuando la adicción al sexo viene acompañada de un consumo reiterado de pornografía, el riesgo de recaídas es mucho mayor (Kingston, Fedoroff, Firestone, Curry y Bradford, 2008).

El problema de los sexoadictos no es tanto la intensidad de sus impulsos como la dificultad para controlarlos o la presencia de motivos no sexuales (soledad, alivio del malestar emocional, dependencia emocional, etcétera) en su conducta sexual. Es ahí donde debe recalar la terapia.

La motivación para el tratamiento es escasa porque la hipersexualidad se tiende a calificar de vicio, no de enfermedad. Por ello, los sujetos afectados tratan de ocultar o minimizar la realidad. Lo que trae al paciente a la consulta habitualmente es el consumo de alcohol o de cocaína o la depresión. Solo después aflora la adicción al sexo, que está enmascarada en otros problemas: conflictos familiares, divorcios, etcétera.

Otras veces lo que motiva al tratamiento son las deudas (casas de masajes, "peep-shows", prostibulos, etcétera) o los problemas familiares. Las mujeres sufren una doble sensación de vergüenza, en función de su rol de protección de la familia, y experimentan un descenso brutal de la autoestima, autorrechazo y depresión.

Hay muchos retos de futuro respecto a este diagnóstico. El conocimiento de la adicción al sexo en la mujer es aún muy limitado. Lo mismo cabe decir de otros aspectos importantes para caracterizar a este cuadro clínico, como los factores de riesgo en el desarrollo evolutivo, la historia familiar, la comorbilidad, los marcadores biológicos o los rasgos de personalidad (Kafka, 2010). Asimismo todavía no se dispone de tratamientos psicológicos basados en la evidencia para hacer frente a este cuadro clínico. Los tratamientos disponibles hasta la fecha son muy variados: psicoterapia individual, terapia de grupo basada 
en el modelo de los doce pasos, farmacoterapia o tratamientos intensivos en régimen de internamiento.

\section{Reconocimientos}

El autor pertenece al Grupo Consolidado de Investigación en Psicología Clínica del Gobierno Vasco y a la UFI 11/04 de la Universidad del País Vasco (UPV/EHU).

\section{Conflicto de intereses}

El autor manifiesta no tener ningún conflicto de intereses que pueda afectar a este artículo.

\section{Referencias}

Alonso-Fernández, F. (1996). Las otras drogas. Madrid: Temas de Hoy.

Bancroft, J., Jannsen, E., Carnes, L., Strong, D.A., Goodrich, D. y Long, J. S. (2004). Sexual activity and risk taking in young heterosexual men: The relevance of sexual arousal, mood and sensation seeking. Journal of Sex Research, 41, 181-192.

Blankenship, R. y Laaser, M. (2004). Sexual addcition and ADHD: Is there a connection? Sexual Addiction \& Compulsivity, 11, 7-20.

Carnes, P. (1983). Out of the shadows: Understanding sexual addiction. Minneapolis, MN: CompCare.

Echeburúa, E. (1999). ¿Adicciones... sin drogas? Las nuevas adicciones (juego, sexo, comida, compras, trabajo, Internet). Bilbao: Desclée de Brouwer.

Echeburúa, E. y Corral, P. (2010). Adicción a las nuevas tecnologias y a las redes sociales. Adicciones, 22, 91-96.

Echeburúa, E., Bravo de Medina, R. y Aizpiri, J. (2005). Alcoholism and personality disorders: An exploratory study. Alcohol \& Alcoholism, 40,323-326.

Echeburúa, E., Bravo de Medina, R. y Aizpiri, J. (2007). Comorbidity of alcohol dependence and personality disorders: A comparative study. Alcohol \& Alcoholism, 42, 618-622.

Echeburúa, E., Bravo de Medina, R. y Aizpiri, J. (2009). Personality disorders among alcohol-dependent patients manifesting or not manifesting cocaine abuse: A comparative pilot study. Substance Use \& Misuse, 44, 981-989.

Goodman, A. (1997). Sexual addiction. En J. H. Lowenson, P. Ruiz, R. B. Millman y J.G. Langrod (Eds.), Substance abuse: A comprehensive textbook ( $3^{\text {rd }}$. ed., pp. 340-354). Baltimore: Williams \& Wilkins.

Goodman, A. (2008). Neurobiology of addiction: An integrative review. Biochemical Pharmacology, 75, 266-322.

Janssen, E., Goodrich, D., Petrocelli, J. V. y Bancroft, J. (2009). Psychophysiological response patterns and risky sexual behavior in heterosexual and homosexual men. Archives of Sexual Behavior, 38, 538-550.

Kafka, M. P. (2010). Hypersexual disorder: A proposed diagnosis for DSM-V. Archives of Sexual Behavior, 39, 377-400.

Kalichman, S. C., Cherry, C., Cain, D., Pope, H. y Kalichman, M. (2005). Psychosocial and behavioral correlates of seeking sex partners on the internet among HIV-positive men. Annals of Behavioral Medicine, 30, 243-250

Kingston, D. A., Fedoroff, P., Firestone, P. Curry, S. y Bradford, J. M. (2008). Pornography use and sexual aggression: The impact of frequency and type of pornography use on recidivism among sexual offenders. Aggressive Behavior, 34, 341-351.

Koob, G. F. (2009). Susbstrates for the neurobiology for the dark side of compulsivity in addiction. Neuropharmacology, 56 (Suppl. 1), 18-31.

Krueger, R. B. y Kaplan, M. S. (2000). Disorders of sexual impulse control in neuropsychiatric conditions. Seminars in Clinical Neuropsychiatry, 5, 266-274.

Langström, N. y Hanson, R. K. (2006). High rates of sexual behavior in the general population: Correlates and predictors. Archives of Sexual Behavior, 35, 37-52.

Miner, M. H., Coleman, E., Center, B. A., Ross, M. y Rosser, B. R. S. (2007). The Compulsive Sexual Behavior Inventory: Psychometric properties. Archives of Sexual Behavior, 36, 579-587.

Nelson, K. G. y Oehlert, M. E. (2008). Psychometric exploration of the Sexual Addiction Screening Test in veterans. Sexual Addiction \& Compulsivity, 15, 39-58.

Potenza, M. (2006). Should addictive disorders include non-substance related conditions? Adicction, 101 (Suppl. 1), 142-151.

Raymond, N. C., Coleman, E. y Miner, M. H. (2003). Psychiatric comorbidity and compulsive/impulsive traits in compulsive sexual behavior. Comprehensive Psychiatry, 44, 370-380.

Reid, R. C. (2007). Assessing readiness to change among clients seeking help for hypersexual behavior. Sexual Addiction \& Compulsivity, 14, 167-186.

Reid, R. C., Harper, J. M. y Anderson, E. H. (2009). Coping strategies used by hypersexual patients to defend against the painful effects of shame. Clinical Psychology and Psychotherapy, 16, 125-138. 
